\title{
Spiritual Care als ärztliche Aufgabe?
}

\section{Simon Peng-Keller}

Prof. Dr. theol., Professor für Spiritual Care an der Universität Zürich und Seelsorger im Kompetenzzentrum Palliative Care des UniversitätsSpitals Zürich

\section{Neues Lehrangebot an der Universität Zürich}

Für Medizinstudierende der Universität Zürich gibt es seit neuestem ein Lehrangebot für Spiritual Care. Die Medizinischen Fakultäten der Universitäten Basel und Bern bieten schon seit einiger Zeit Lehrveranstaltungen an, die spirituelle Aspekte der ärztlichen Tätigkeiten berühren. Dies entspricht den Vorgaben des Schweizerischen Lernzielkatalogs für medizinische Ausbildung (SCLO), wonach Medizinstudierende im Hinblick auf ihre künftige Berufstätigkeit lernen sollen, neben kulturellen und sozialen Faktoren auch spirituelle und religiöse Aspekte zu beachten. Der von der Joint Commission of the Swiss Medical Schools erstellte SCLO sieht vor, dass alle Ärzte und Ärztinnen, unabhängig von ihrer Spezialisierung, einer solchen Grundkompetenz bedürfen.

Die offenen Formulierungen des SCLO lassen viel Raum zur konkreten Ausgestaltung der entsprechenden Lehre. Im Vergleich zu dem, was angehende Ärztinnen und Ärzte zu erlernen haben, scheint die Erweiterung der psychosozialen Behandlungsaspekte um die religiös-spirituelle Dimension lediglich eine moderate Anpassung an die internationale Entwicklung zu sein. Dennoch handelt es sich dabei um einen nicht unerheblichen Schritt, der das ärztliche Selbstverständnis berührt. Ein solcher Schritt ist begründungsbedürftig.

\section{Spiritual Care comme devoir médical?}

Au vu des nouvelles offres de cours dans la formation de base des médecins, cet article tente de répondre à la question de savoir si le Spiritual Care doit être envisagé comme une tâche médicale. Etant donné que les exigences requises pour devenir médecin sont déjà très élevées, l'on peut se demander s'il est judicieux d'étendre les compétences en y incluant le Spiritual Care. Contrairement à l'idée selon laquelle cela ne représenterait qu'une charge de plus pour les médecins, l'auteur incite à la réflexion en faisant valoir qu'en l'occurrence, il ne s'agit pas tant d'une tâche supplémentaire que d'un aspect fondamental d'une prise en charge médicale axée sur le patient. Dans la mesure où les approches et les pratiques religieuses et spirituelles ont une influence sur notre manière d'appréhender la maladie et sur les décisions concernant la fin de vie, elles doivent être prises en compte dans les soins médicaux. Le Spiritual Care n'est pas un fardeau de plus, mais un nouvel outil pour appréhender les tâches qui font partie de la fonction médicale depuis toujours, et qu'il s'agit d'intégrer en tant qu'attitude à la base de toute activité médicale.

\section{Dass Ärzte Patienten ermutigen und trösten und manchmal einen Seelsorger beiziehen, hat eine lange Tradition.}

Epochen der abendländischen Geistes- und Medizingeschichte die Aufgaben des Arztes und des Priesters nahe beisammen gewesen sein: Gehört es nicht zu den bedeutsamen Errungenschaften der Moderne, dass sich das medizinische, psychotherapeutische und seelsorgliche Tätigkeitsfeld ausdifferenziert und professionalisiert hat?

Es braucht also gute Gründe, um das ärztliche Aufgabenspektrum unter den gegenwärtigen Bedingungen auszuweiten. Zu erwägen sind insbesondere die folgenden Aspekte:

1. Orientiert man sich am Ideal einer patientenzentrierten Gesundheitsversorgung, so wie sie alle medizinischen Fakultäten und Gesundheitsinstitutionen der Schweiz einfordern, kommt Ärztinnen und Ärzten die Aufgabe zu, religiöse und spirituelle Aspekte dann in den Blick zu nehmen, wenn diese für ihre Patientinnen und Patienten wichtig sind. Um dies in professioneller Weise tun zu können, braucht es eine angemessene Ausbildung.

2. Es ist empirisch gut belegt, dass religiöse und spirituelle Haltungen und Praktiken den Umgang mit Krankheit sowie Entscheidungen am Lebensende beeinflussen. Dabei hat sich auch gezeigt, dass die diesbezüglichen Einstellungen der behandelnden Ärztinnen und Ärzte einen nicht zu vernachlässigenden therapeutischen Faktor darstellen.

3. Kaum bestritten ist, dass spirituelle und religiöse Fragen sowie Bewältigungsformen besonders in Situationen wichtig werden, in denen kurative medizinische Möglichkeiten ausgeschöpft sind. Da 
Ärztinnen und Ärzte unabhängig von ihrer Spezialisierung mit solchen Situationen konfrontiert werden, bedürfen sie, um professionell handeln zu können, einer Grundkompetenz in Palliative Care. Dazu gehört nicht zuletzt auch die Berücksichtigung von spirituellen und religiösen Bedürfnissen und Überzeugungen. In einer jüngst von Dr. med. Klaus Bally im Rahmen des Nationalen Forschungsprogramms «Lebensende» (NFP 67) durchgeführten Umfrage waren gegen $60 \%$ der befragten Schweizer Hausärzte der Ansicht, dass ein kompetenter Umgang mit spirituellen Bedürfnissen eines Patienten ein wichtiges bis sehr wichtiges Qualitätsmerkmal einer guten palliativmedizinischen Versorgung darstellt. Nur 38\% von ihnen fühlen sich allerdings im Umgang mit spirituellen Bedürfnissen ihrer Patienten sicher.

4. Nicht zuletzt gibt es gesundheitspolitische Gründe, angehende Ärztinnen und Ärzte in die Anliegen und Aufgaben einer Spiritual Care einzuführen. So stellt beispielsweise die Zuwanderung von kriegstraumatisierten Menschen mit anderen kulturellen und religiösen Hintergründen für das schweizerische Gesundheitswesen eine Herausforderung dar, zu deren Bewältigung es auch ein Verständnis für fremde religiöse Überzeugungen und Praktiken braucht. Die von der UNHCR 2015 publizierte Review for Mental Health and Psychosocial Support Staff Working with Syrians Affected by Armed Conflict gibt dazu wichtige Hinweise.

\section{Nur für spezialisierte Fachleute?}

Man könnte einwenden, dass es sich bei dem Genannten um zweifellos wichtige Aspekte der Gesundheitsversorgung handelt, diese jedoch - von Extremsituationen abgesehen - besser von spezialisierten Fachleuten beurteilt und wahrgenommen werden sollten, statt sie Ärztinnen und Ärzten aufzubürden. Bis zu einem

Es ist empirisch gut belegt, dass religiöse und spirituelle Haltungen und Praktiken den Umgang mit Krankheit beeinflussen.

gewissen Grad ist dieser Einwand sicherlich zutreffend. Zur ärztlichen Professionalität gehört das Wissen um die Grenzen des eigenen Könnens. Ärztinnen und Ärzte können neben ihren medizinischen Aufgaben nicht auch noch jene der Seelsorge wahrnehmen. Dass sie in ihrem ärztlichen Tun die spirituellen und religiösen Aspekte jedoch dann mitberücksichtigen, wenn diese für die Patientinnen und Patienten bedeutsam sind, darf aus den genannten Gründen erwartet werden. Spirituelle Ressourcen zeigen sich allerdings meist erst dann, wenn Patientinnen und Patienten aktiv darauf angesprochen werden.

Schliesslich bedarf auch die Vorstellung, der ärztlichen Profession werde im Zusammenhang von Spiritual Care eine zusätzliche «Bürde» auferlegt, einer näheren Überprüfung. Genauer besehen geht es nicht um zusätzliche Pflichten, sondern um den Erwerb von spe-

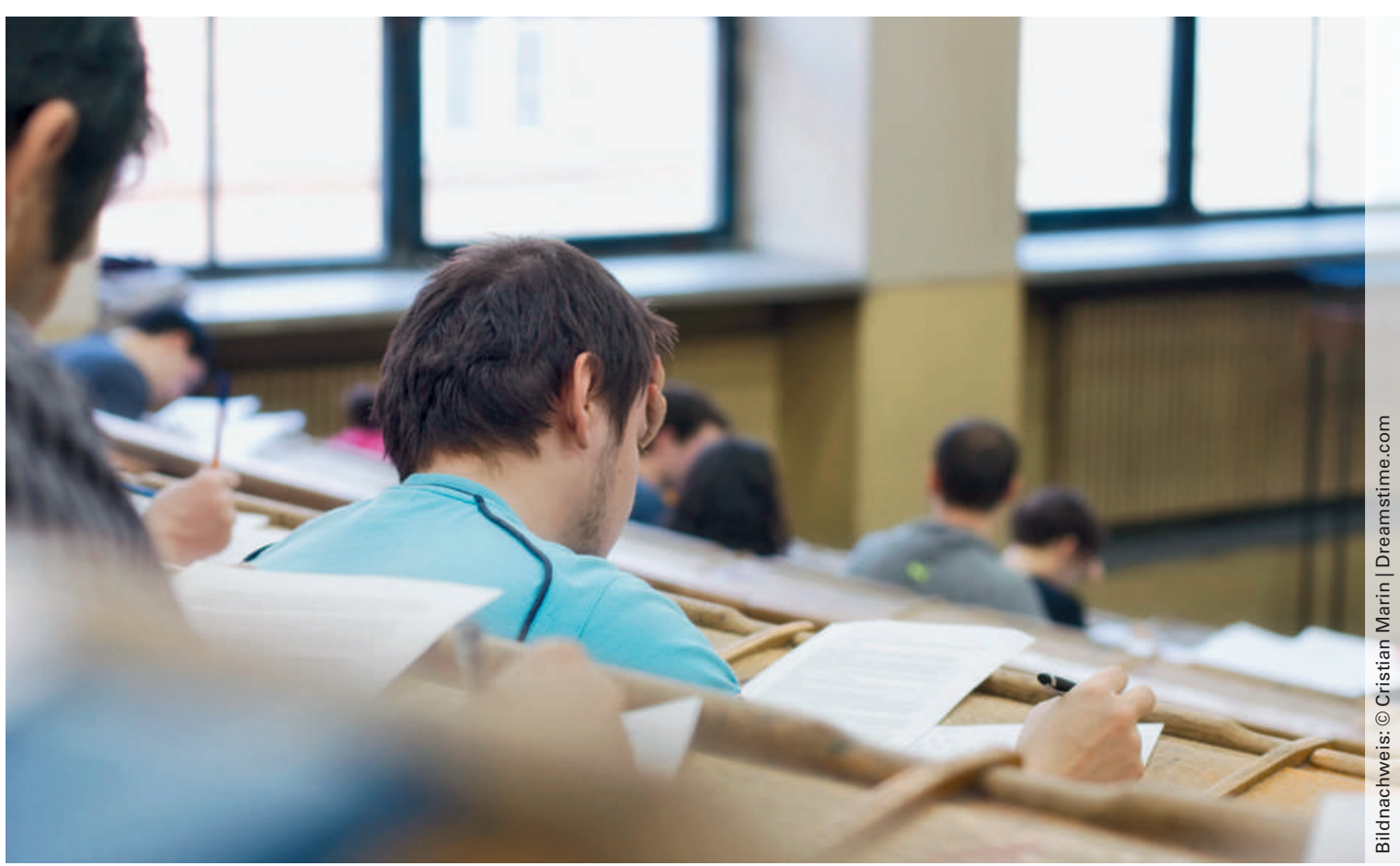

An der Universität Zürich gibt es seit kurzem ein neues Lehrangebot für Spiritual Care. 
zifischen Kompetenzen im Umgang mit bereits bestehenden Aufgaben. In seiner Berufstätigkeit in Situationen zu kommen, auf die man nicht hinreichend vorbereitet wurde, ist frustrierend. Oder positiv formuliert: Die Arbeitszufriedenheit von Ärztinnen und Ärzten erhöht sich, wenn sich die Patientenzufriedenheit erhöht, und diese nimmt nachweislich zu, wenn in der ärztlichen Behandlung auch spirituell-religiöse Aspekte berücksichtigt werden. Faktisch wird das von vielen Ärztinnen und Ärzten auch bereits gemacht.

\section{Länderspezifische Unterschiede}

Eine in Holland, Belgien, Spanien und Italien durchgeführte Studie (Evans et al. 2014) brachte allerdings deutliche länderspezifische Unterschiede ans Licht: Während 37\% der holländischen Hausärzte, die an der Studie teilnahmen, mit ihren lebensbedrohlich kranken Patienten über spirituelle und existenzielle The-

In seiner Berufstätigkeit in Situationen zu kommen, auf die man nicht hinreichend vorbereitet wurde, ist frustrierend.

men sprachen, ist der Anteil bei ihren italienischen und spanischen Kollegen viel geringer (9\% bzw. 7\%). Nach K. Ballys Studie dürften die Schweizer Hausärzte diesbezüglich näher bei ihren nördlichen als bei ihren südlichen Kollegen liegen. Zu vermuten ist, dass spanische und italienische Ärzte noch stärker von der Vorstellung geleitet sind, dass Patienten entweder bekennende Katholiken sind, für die katholische Geistliche zuständig sind, oder aber säkulare Bürger/-innen, die keines spirituellen Beistands bedürfen. In den Niederlanden hingegen haben alle Patientinnen und Patienten, unabhängig von ihrer religiösen (Nicht-)Zugehörigkeit, einen rechtlich verbürgten Anspruch auf Spiritual Care, wenn sie länger als 24 Stunden in einem Krankenhaus sind. Dies führt dazu, dass diese Aufgabe stärker interprofessionell wahrgenommen wird.

Ob in Italien, Holland oder der Schweiz: Als zentrale Entscheidungsträger und -moderatoren spielen Ärztinnen und Ärzte eine Schlüsselrolle, wenn es um den Einbezug spezialisierter Formen spiritueller und religiöser Unterstützung und deren Gewährleistung im klinischen Kontext geht. Dass Ärzte Patienten ermutigen und trösten und manchmal einen Seelsorger beiziehen, hat eine lange Tradition. Insofern ist Spiritual Care ein neues Wort für eine alte und nach wie vor wichtige Aufgabe unter den Bedingungen heutiger Medizin. Zu diesen Bedingungen gehört die Ausdifferenzierung der professionellen Aufgaben ebenso wie eine Kompetenz zur interprofessionellen Zusammenarbeit und ein neuer Sinn für die nicht-medizinischen Aspekte ärztlicher Praxis.

Erhard Taverna beschloss vor vier Jahren seine kritische Auseinandersetzung mit dem «Mehrwert 'Spiritualität'» mit einer lateinischen Weisheit: «Vielleicht genügt es, von einer persönlichen, sinnstiftenden Grundeinstellung zu sprechen, die religiöses Denken beinhalten kann, aber nicht muss. Der alte Spruch: Medicus curat, natura sanat, Deus salvat kommt diesem Zusammenspiel physischer, psychischer und geistiger Voraussetzungen vielleicht immer noch am nächsten" (SÄZ 2012;93(45):1678). Man könnte dies als Plädoyer für eine ärztliche Selbstbescheidung auf die kurative Aufgabe lesen. Doch sieht sie Taverna im Zusammenspiel, in dem es auch um sinnstiftende Grundeinstellungen geht, die mitunter einen religiösen Charakter haben, wie das bei Taverna selbst offenbar der Fall ist. Übersetzt man diesen von einer religiösen Gewissheit geprägten Spruch in die Sprache heutiger Spiritual Care, so besteht die Ärztinnen und Ärzten zumutbare Spiritual Care darin, ihre ärztliche Aufgabe so wahrzunehmen, dass die sinnstiftenden Grundeinstellungen von Patienten in die Care einbezogen und soweit als möglich gestärkt werden.

\section{Literatur}

Evans N, Costantini M, Pasman HR, Van den Block L, Donker GA, Miccinesi G, et al. End-of-life communication: a retrospective survey of representative general practitioner networks in four countries. Journal of pain and symptom management. 2014;47(3):604-19.

Die Antrittsvorlesung von Professor Peng-Keller findet am 25.4.16 um 18.15 Uhr in der Aula der Universität Zürich statt. Thema: Spiritual Pain. Annäherung an einen Schlüsselbegriff heutiger Spiritual Care. 